\title{
Tuberculosis, los errores que cometemos. Experiencia del Hospital San Juan de Dios
}

\author{
ANGÉLICA BELLO F.*, CARLOS CARRASCO R.*, CARMEN ANDRADE V.* y VICTORINO FARGA C.**
}

\section{Tuberculosis, our mistakes. The experience at San Juan de Dios Hospital}

Tuberculosis is an infectious disease that in Chile is far from eradicated. To ask what improvements we could make, we must recognize from our experience at San Juan de Dios Hospital, the major mistakes which many of us do, both in the diagnosis and management of the disease. Possibly the most important, common and serious mistake, is to believe that tuberculosis is eradicated, because if we believe it does no longer exists, then we do not seek it, and leave undiagnosed infectious cases, keeping the reservoirs of the disease.

Key words: Tuberculosis; disease management; communicable diseases; Chile.

\section{Resumen}

La tuberculosis es una enfermedad infecciosa que en Chile está lejos de ser eliminada. Para plantear qué mejoras podríamos realizar para su control es necesario conocer los principales errores que según nuestra experiencia, en el Hospital San Juan de Dios, muchos de nosotros cometemos, tanto en el diagnóstico como en el manejo de la enfermedad. El más común y grave, posiblemente sea el de creer que la tuberculosis está erradicada, porque si creemos que ya no existe, no la buscaremos y dejaremos casos sin diagnosticar, manteniendo los reservorios que trasmiten la enfermedad.

Palabras clave: Tuberculosis; manejo de enfermedades; enfermedades transmisibles; Chile.

\section{Introducción}

La tuberculosis (TBC) es una enfermedad infecciosa que en el año 1993 fue declarada por la Organización Mundial de la Salud (OMS) una Emergencia Global por su alta incidencia y mortalidad $^{1}$. Se estima que anualmente enferman nueve millones de personas y de estas mueren alrededor de dos millones. En Chile, el año 2013, fallecieron 275 personas de $\mathrm{TBC}^{2}$, pese a ser una enfermedad curable.

Por este motivo, en el Servicio de Enfermedades Respiratorias del Hospital San Juan de Dios, del Servicio Metropolitano Occidente, nos ha parecido importante revisar, a partir de nuestra experiencia como centro que trata anualmente alrededor de 200 pacientes con esta patología, cuáles son los principales errores que cometemos diariamente.

\section{Primer error: creer que la tuberculosis es una enfermedad erradicada}

Actualmente la incidencia de TBC en Chile es de 14 casos por 100.000 habitantes, cifra que se ha mantenido estable en los últimos 5 años ${ }^{2}$, alejada de los pronósticos favorables que existían en el pasado, donde se esperaba que el año $2010 \mathrm{su}$ incidencia fuera menor a 10/100.000 habitantes, es decir hubiera alcanzado la etapa de eliminación avanzada, meta propuesta por la Organización Mundial de la Salud (OMS) ${ }^{3}$.

En la Figura 1 puede observarse como se ha

* Médicos del Servicio de Enfermedades Respiratorias Hospital San Juan de Dios. Santiago, Chile.

** Médico Asesor del Ministerio de Salud, Gobierno de Chile. 


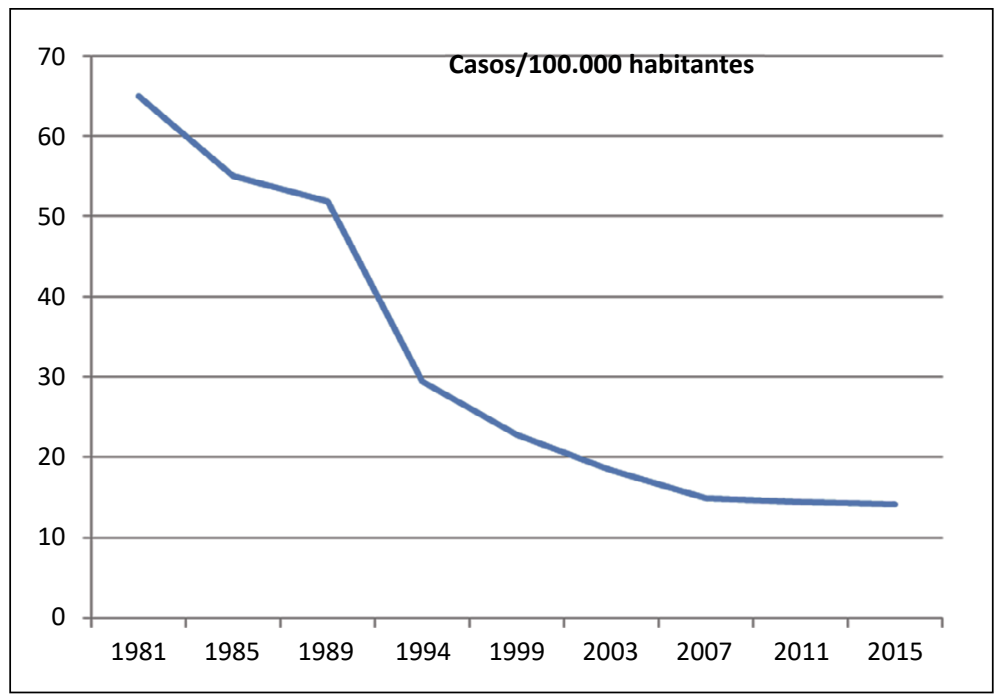

Figura 1. Morbilidad de TBC en Chile 1981-2015. Morbilidad expresada en casos/100.000 habitantes. Fuente: PROCET. enlentecido el descenso en la incidencia de la TBC en distintos períodos de tiempo, en los cuales se limitaron las acciones del Programa de Control y Eliminación de la Tuberculosis (PRO$\mathrm{CET})^{3,4}$. Es más, según datos del último Informe de Situación TBC del Ministerio de Salud, en 2015 hubo un aumento de la incidencia de nuevos casos, lo que rompe la tendencia al descenso que se observaba anteriormente.

Este retroceso en el control de la TBC se puede explicar por variadas causas, pero es posible que una de las más importantes sea que dejamos de pensar en la TBC, la eliminamos de nuestras posibilidades diagnósticas al enfrentar a un paciente. Sin embargo, actualmente la mayoría de los casos de TBC se dan en población joven, es decir, corresponden a casos nuevos aparecidos en la comunidad por contagios recientes; no son reactivaciones de tuberculosis latentes, como en los países que están en vías de eliminar la enfermedad.

Otra muestra de que estamos lejos de eliminar la $\mathrm{TBC}$ en Chile es que los casos pulmonares con baciloscopia positiva (formas contagiosas) son aún muy frecuentes. El año 2013 se logró el mayor descenso de estas formas a 8,1/100.000, pero el 2014 éstas aumentaron a 8,3/100.000 y el 2015 la cifra es aún mayor, 9/100.000 habitantes, revirtiendo su tendencia al descenso de los años precedentes $^{2}$.

Por otra parte, cuando revisamos la incidencia de la TBC por regiones de nuestro país, constatamos que existen grandes diferencias en las tasas, con una mayor concentración de casos en la zona norte de Chile (Figura 2).

Lo mismo ocurre en la Región Metropolitana, que tiene una incidencia de TBC de 14,3/100.000, cercana al promedio nacional. Sin embargo, al analizar las cifras por Servicio de Salud se encuentra una importante heterogeneidad en la distribución de la enfermedad, seguramente debida a las diferencias socioeconómicas existentes dentro de esta región. Por ejemplo, en la Región Metropolitana existen tasas muy dispares entre el Servicio de Salud Norte con 21,4/100.000, el Servicio de Salud Occidente con 14,1/100.000, similar al promedio nacional y el Servicio de Salud Oriente con $8,3 / 100.000$, una de las más bajas del país.

\section{Segundo error: baja solicitud de baciloscopias (BK)}

Esta es actualmente la falla más importante en la aplicación del PROCET ${ }^{4,5}$.

La tuberculosis pulmonar, forma más frecuente de $\mathrm{TBC}$, aún se diagnóstica mayoritariamente por BK positiva $(77 \%)^{2}$, es decir en pacientes con alta carga bacilar. Si consideramos que la BK es un método diagnóstico de la $\mathrm{TBC}$ gratuito, rápido $\mathrm{y}$ de fácil disponibilidad y que, además, es capaz de evidenciar las formas de tuberculosis más contagiosas, que son las de mayor importancia epidemiológica, resulta incomprensible la baja solicitud de este examen en todos los niveles de atención médica, sobre todo a nivel primario, donde deberían pesquisarse de preferencia y más precozmente estos pacientes. En el Servicio de Salud Occidente, dos tercios de los casos de TBC se diagnostican en el nivel secundario y terciario, indicando un bajo nivel de pesquisa en la atención primaria. Entre los pacientes cuya 


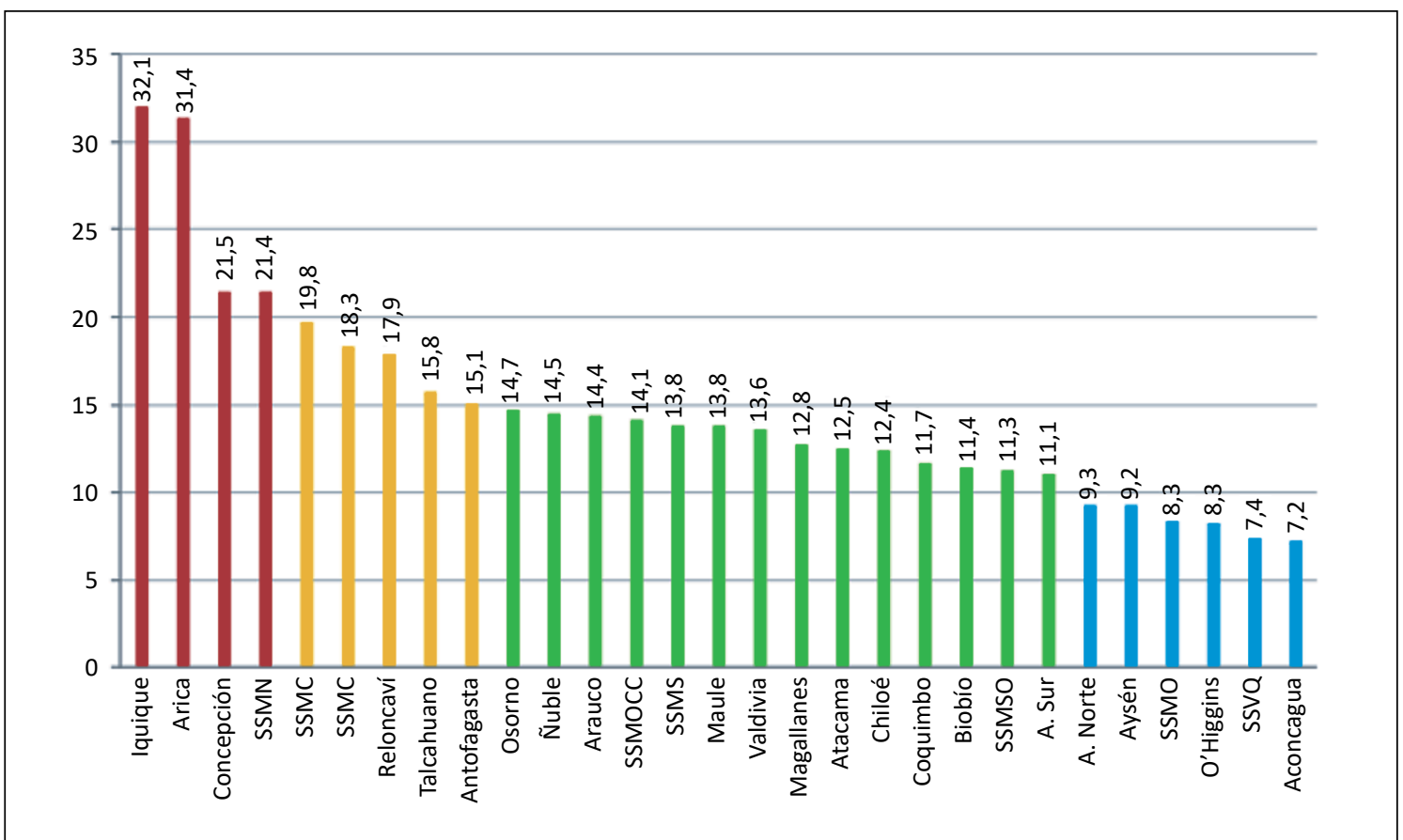

Figura 2. Incidencia de tuberculosis en Chile por Servicio de Salud. Incidencia expresada en casos/100.000 habitantes. SSM= Servicio de Salud Metropolitano: N=Norte; S=Sur; SO= Sur-Oriente; C= Centro; OCC= Occidente; $\mathrm{O}=$ Oriente. SSVSA= Servicio de Salud Valparaíso-San Antonio. SSVQ= Servicio de Salud Viña del Mar-Quillota. A. Sur= Araucanía Sur. A. Norte= Araucanía Norte. Fuente: Informe Situación Tuberculosis 2015, PROCET.

primera consulta se realiza en el sistema privado, la sospecha diagnóstica es aún menor, generando un mayor retraso en el diagnóstico.

Al revisar las cifras sobre la solicitud de BK en los policlínicos del Hospital San Juan de Dios, esta se concentra sólo en las subespecialidades de Enfermedades Respiratorias e Infectología, lo que indica un bajo nivel de sospecha de la enfermedad en el resto de los departamentos.

\section{Tercer error: no considerar las poblaciones de riesgo}

En los últimos años se observa una mayor concentración de enfermos de TBC en territorios y poblaciones de riesgo. En el último informe emitido por el PROCET respecto a la situación del año $2015^{2}$ es posible observar que el $46 \%$ de los casos de TBC pertenece a algún grupo de riesgo, es decir, se trata de pacientes que presentan alguna condición que genera un riesgo de desarrollar la enfermedad. Conocer estas condiciones es importante para focalizar la pesquisa en estos grupos $^{4,5}$ (Tabla 1).

Respecto a los grupos de riesgo identificados
Tabla 1. Factores de riesgo en pacientes con TBC

\begin{tabular}{|lc|}
\hline Grupo de Riesgo & $\begin{array}{c}\text { Porcentaje del } \\
\text { total de TBC } \\
(\%)\end{array}$ \\
\hline $\begin{array}{l}\text { Extranjeros (de países con alta } \\
\text { prevalencia) }\end{array}$ & 10,1 \\
\hline Alcoholismo & 9,3 \\
\hline Co-infección VIH & 8,4 \\
\hline Drogadicción & 6,1 \\
\hline Diabetes Mellitus & 6 \\
\hline Contacto de TBC & 4 \\
\hline Población indígena & 3,2 \\
\hline Otras inmunodepresiones (no VIH) & 2,7 \\
\hline Población privada de libertad & 2,5 \\
\hline Situación de calle & 2,3 \\
\hline Personal de salud & 0,8 \\
\hline
\end{tabular}

Fuente: Informe Situación Tuberculosis 2015. PROCET.

en los últimos años ${ }^{6,7}$, la infección por VIH corresponde a un $8 \%$ de los enfermos con TBC. Se estima que los infectados VIH tienen 20 veces más riesgo de reactivación de una TBC latente, 
siendo la TBC la principal causa de muerte infecciosa en este grupo de pacientes. Además, La TBC en los infectados VIH deteriora aún más su estado inmunológico. En estos enfermos se ha descrito una mayor frecuencia de presentaciones clínicas inhabituales, lo que dificulta el diagnóstico.

Otro grupo de riesgo lo constituyen los diabéticos. Según lo establecido por la OMS, los diabéticos tienen un riesgo 2 a 3 veces mayor de desarrollar TBC. Recién este año se incluye la diabetes en el Informe Anual de Tuberculosis 2015 del MINSAL, calculándose en 6\% los pacientes con TBC que presentan diabetes ${ }^{8,9}$. Es posible que este número pueda ser mayor y que exista un importante sub-registro de esta patología.

Los inmigrantes de países de alta prevalencia constituyen otro grupo de importancia. Muchas veces viven en condiciones de hacinamiento, con dificultades o temor al acceso a servicios de salud, dado que frecuentemente se encuentran en condiciones migratorias irregulares. Es importante recalcar que el PROCET asegura el diagnóstico y tratamiento gratuitos de la TBC independiente de la condición migratoria y que es tarea de todos nosotros buscar nuevas formas de abordar este grupo de pacientes, muy diversos culturalmente, para asegurar un diagnóstico precoz y disminuir la transmisión de la infección ${ }^{10,11}$.

En el Hospital San Juan de Dios este año se está realizando un trabajo en conjunto con los policlínicos de mayor riesgo (Infectología, Reumatología, Diabetes y Gastroenterología) para aumentar la solicitud de estudios bacteriológicos en los enfermos sintomáticos respiratorios.

\section{Cuarto error: no solicitar examen VIH en los pacientes con diagnóstico de TBC}

La norma del PROCET indica que en todos los enfermos con diagnóstico de TBC debe realizarse un examen $\mathrm{VIH}^{2,6}$. Esto es fundamental, no sólo porque implica aumentar la pesquisa de infección VIH y, por lo tanto, ofrecer tratamiento retroviral oportuno, sino porque cuando exista co-infección $\mathrm{VIH}$ el tiempo de terapia antituberculosa que recibirá el paciente deberá prolongarse a 9 meses para asegurar su curación ${ }^{6,13}$.

Cuando se revisan los datos chilenos ${ }^{2,6}$ es posible ver que desde el 2011 a la fecha ha existido un aumento en el porcentaje de test de VIH efectuados en los pacientes con diagnóstico de TBC; sin embargo, estamos lejos de la cobertura esperada de $100 \%$ (Figura 3 ).

Al revisar el examen de VIH en los pacientes TBC del Servicio de Salud Metropolitano Occidente, el número de casos con co-infección TBC-VIH ha aumentado entre 2014 y 2015 de $10 \%$ a $15 \%$. Sin embargo, el porcentaje de test de VIH realizados en los enfermos con TBC aún es muy bajo y, lo que es peor, entre 2014 y 2015 este porcentaje disminuyó de 43 a $41 \%$. Esto ha planteado la necesidad de buscar nuevas estrategias para conseguir un aumento de la petición de este examen, lo que se está implementando a partir de este año.

\section{Quinto error: no conocer las últimas modificaciones del PROCET 2014}

Los cambios a la Normas del PROCET generados el año 2014 tuvieron como objetivo sim-

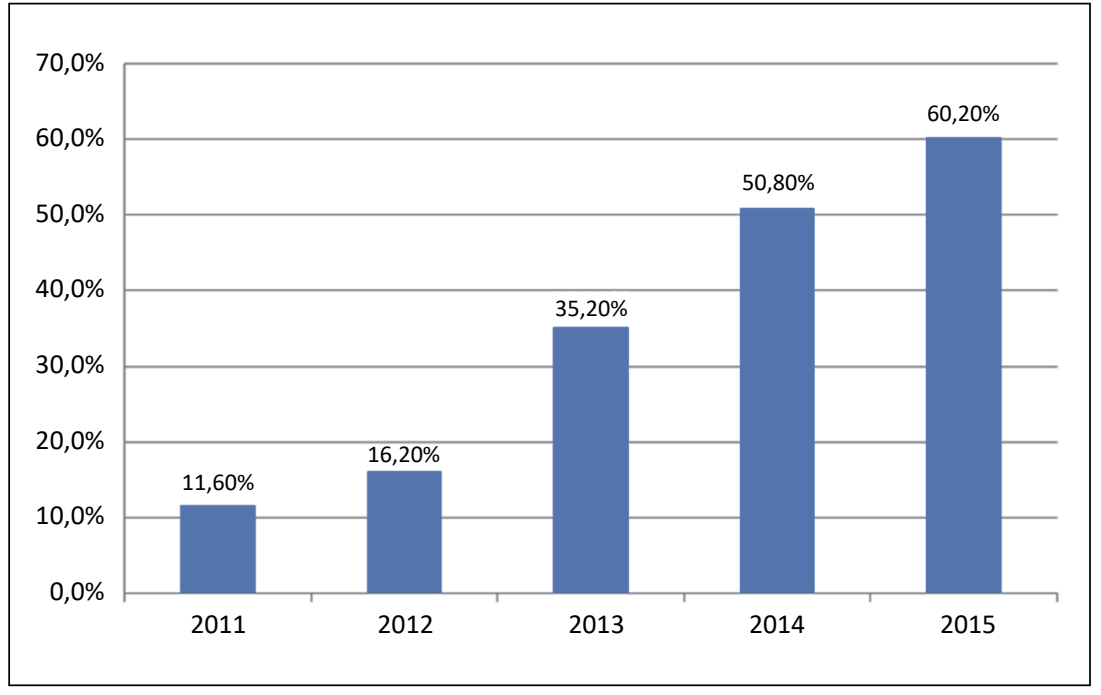

Figura 3. Porcentaje de solicitud de test para VIH entre los casos de TBC. Fuente: Informe Situación Tuberculosis 2015. PROCET. 
plificar y estandarizar el tratamiento actual de la $\mathrm{TBC}^{1,7}$. Si bien esto se lleva a cabo en su mayor parte en la atención primaria, es responsabilidad de todos los médicos saber las modificaciones más importantes, dentro de las cuales se encuen$\operatorname{tran}^{14}$ :

1. Un solo esquema primario unificado, independiente de si el paciente ha sido antes tratado o si tiene confirmación bacteriológica.

2. Cambio de la segunda fase de tratamiento de 2 a 3 veces a la semana.

3. Estudio de susceptibilidad a todos los pacientes, independiente de si presentan alguna condición de riesgo para tener resistencia a algún fármaco.

4. Disminución de la dosis diaria de etambutol de 1.200 a $800 \mathrm{mg}$ para disminuir el riesgo de neuritis óptica.

5. Tratamiento con dosis fijas combinadas de medicamentos.

6. Prolongación del tratamiento a 9 meses en casos especiales (co-infección TBC-VIH, formas cavitarias extensas y mantención de $\mathrm{BK}$ positiva al segundo mes de tratamiento).

La alta tasa de rotación en los equipos encargados de TBC, sobretodo a nivel primario de salud, hace fundamental la capacitación continua del personal para evitar errores en la aplicación de las nuevas Normas del PROCET.

\section{Sexto error: no proteger a las poblaciones de riesgo con quimioprofilaxis}

La quimioprofilaxis es la administración de medicamentos antituberculosos a personas con riesgo de desarrollar TBC para prevenir su aparición ${ }^{1,13}$. Sus principales indicaciones son:

1. Recién nacidos de madre con TBC pulmonar activa.

2. Contactos menores de 15 años de pacientes con TBC pulmonar.

3. Portadores de lesiones radiológicas inactivas de origen tuberculoso.

4. Pacientes con distintos grados de inmunosupresión (VIH, drogas biológicas, etc).

Las personas definidas como portadoras de una TBC latente por tener un PPD positivo o un IGRA (ensayo de liberación de interferón gama) reactivo, con alguna condición de inmunosupresión, deben recibir, siempre que no tengan alguna contraindicación, quimioprofilaxis con isoniacida
$5 \mathrm{mg} / \mathrm{kg}$, sin superar los $300 \mathrm{mg}$ diarios, durante 6 a 9 meses. Se trata de un tratamiento autoadministrado, gratuito, de retiro quincenal de la isoniacida y sujeto a controles tanto clínicos como de laboratorio.

Los principales errores que hemos detectado en la indicación de quimioprofilaxis son:

- Falta de descarte inicial de TBC activa.

- Falta de estudios previos que indiquen una TBC latente (PPD, IGRAs).

- Falta de controles de la terapia, con el riesgo de desarrollar hepatitis graves.

- Mantención de la quimioprofilaxis por tiempos excesivos (a veces por años).

\section{Séptimo error: desconocer la realidad de nuestros pacientes}

La única forma de adentrarse en profundidad en el problema actual de la TBC es conocer qué está ocurriendo con nuestra población enferma, cómo está cambiando el perfil epidemiológico de nuestros pacientes, cual es el medio y la realidad en la que están insertos, para prevenir uno de los problemas actuales, el abandono del tratamiento antes del tiempo necesario para asegurar la curación.

En un estudio publicado en $2015^{15}$ donde se analizaron las auditorías de abandono, se encontró el escaso cumplimiento que tienen estos estudios, lo cual entorpece el análisis de los datos. La mayoría de los pacientes que abandonaban eran hombres jóvenes pertenecientes a algún grupo de riesgo, principalmente alcoholismo y drogadicción. Esto refuerza la importancia de aplicar el "puntaje de abandono" al inicio del tratamiento de los enfermos para establecer estrategias preventivas. Se constató que el abandono o pérdida de seguimiento, ocurre en cualquier fase del tratamiento. Dentro de las causas de abandono se registra el hecho de "sentirse bien", lo que demuestra una falta de conocimiento por parte del paciente de la importancia de mantener el tratamiento hasta su curación.

Son muchas las medidas que pueden tomarse para prevenir los abandonos. Por ejemplo, recientemente en mayo de 2016 se inició un Plan Piloto en la comuna de Recoleta, para asegurar el diagnóstico oportuno y la continuidad del tratamiento basados en un "Programa de Acompañamiento" en pacientes en situación de calle.

Los enfermos que abandonan el tratamiento mantienen las fuentes de contagio en la comunidad, limitando la eficacia del Programa de Control y Eliminación de la Tuberculosis. 


\section{Conclusiones}

a) Lamentablemente la TBC en Chile está lejos de estar erradicada. Esto es evidente al revisar los datos nacionales donde aún la mayoría de casos se diagnostica en población joven, con aumento de las formas pulmonares con BK positiva, que son las formas más contagiosas.

b) Existe una baja solicitud de BK en los niveles de atención más básicos, lo que ha generado un aumento de diagnósticos tardíos en los niveles de atención más complejos, produciendo un mayor gasto en salud y persistencia del contagio en la comunidad.

c) La heterogeneidad existente en la incidencia tanto entre las diferentes regiones de nuestro país como dentro de las diferentes áreas en Santiago, demuestra realidades dispares que debemos ser capaces de identificar para implementar nuevas estrategias que logren la detección oportuna y el manejo adecuado de los enfermos.

d) Es importante reconocer los pacientes con factores de riesgo de desarrollar TBC para realizar una pesquisa activa en ellos; del mismo modo y según las normas existentes, debemos identificar a los enfermos con TBC latente que requieran quimioprofilaxis.

Finalmente, dentro de los desafíos que plantea el manejo de la TBC, está el de hacer diagnósticos más oportunos para disminuir las inaceptables cifras de letalidad debidas a diagnósticos tardíos que aún tiene la TBC en Chile e identificar precozmente a los pacientes con riesgo de abandono de tratamiento, incorporando estrategias de prevención que disminuyan los focos de infección en la comunidad.

En síntesis, las principales fallas actuales para el control de la TBC en Chile serían:

- Localización de casos (pesquisa) baja y no focalizada en grupos de riesgo.

- Altas cifras de letalidad y pérdidas de seguimiento de los enfermos (abandonos).

- Insuficiente estudio de los contactos de enfermos contagiosos.

- Debilitamiento del Programa de Control de la Tuberculosis en los últimos años.

Es probable que los errores detectados en el Hospital San Juan de Dios sean, en menor o mayor grado, un reflejo de las dificultades y desafíos que plantea la lucha contra la tuberculosis a nivel nacional.

\section{Bibliografía}

1.- RODRÍGUEZ J. Tuberculosis Latente. Rev Chil Enf Respir 2012; 28: 61-8.

2.- PROCET. Tuberculosis, Informe Situación Chile 2014.

3.- HERRERA T, FARGA V. Historia del Programa de Control de la Tuberculosis de Chile, Rev Chil Enferm Respir 2015; 31: 227-31.

4.- PEÑA C, FARGA V. Avanzando en la lucha contra la tuberculosis en Chile. Rev Chil Enferm Respir 2014; 29: 219-27.

5.- PEÑA C, FARGA V. El difícil camino del control sanitario de la tuberculosis. Rev Chil Enferm Respir 2012; 28: 311-8.

6.- GARCÍA C. Tuberculosis en grupos de riesgo en la Región Metropolitana. 2008. Rev Enferm Respir 2010; 26: 105-11.

7.- PROCET. Manual de Organización y Procedimientos del Programa Nacional de Control y Eliminación de la Tuberculosis 2014.

8.- HERRERA T, LEIVA E, MARTÍN F, MIRANDA M, MORALES C. Asociación entre tuberculosis y diabetes mellitus en la Región Metropolitana. Rev Chil Enferm Respir 2013; 29: 171-5.

9.- RAMONDA P, PINO P, VALENZUELA L. Diabetes mellitus como factor predictor de tuberculosis en el Servicio de Salud Metropolitano Sur en Santiago, Chile. Rev Chil Enferm Respir 2012; 28: 277-85.

10.- YÁÑEZ A. Tuberculosis en inmigrantes. Situación Chile- Perú. Rev Chil Enferm Respir 2010; 26: 161-4.

11.- PEÑA C, CAAMAÑO R, MESA M, URZÚA R, PINOCHET M, MIRANDA C. Aporte de los inmigrantes a la tuberculosis en un Servicio de Salud de Chile. Rev Chil Enferm Respir 2016; 32: 41-9.

12.- PEÑA C, CÉSPED M, WOLFF M, ÁLVAREZ F, GARAY C, MEDINA M, et al. Diagnóstico bacteriológico de tuberculosis pulmonar mediante fibrobroncoscopía en pacientes VIH. Rev Chil Enferm Respir 2014; 30: 46-53.

13.- FARGA V, CAMINERO J A. Tuberculosis. Tercera Edición. Editorial Mediterráneo. 2011.

14.- FARGA V. Cambios en el tratamiento de la tuberculosis, Rev Chil Enferm Respir 2014; 30: 174-9.

15.- TORRES Z, HERRERA T. Perfil del paciente con tuberculosis que abandona el tratamiento en Chile. Rev Chil Enferm Respir 2015; 31: 52-7.

Correspondencia a:

Dra. Angélica Bello Fernández.

Servicio de Enfermedades Respiratorias,

Hospital San Juan de Dios. Huérfanos 3255,

Santiago, Región Metropolitana, Chile.

Email: angebf19@gmail.com 